\title{
Existence of an invariant measure for stochastic evolutions driven by an eventually compact semigroup
}

\author{
JORIS BIERKENS, ONNO VAN GAANS AND SJOERD VERDUYN LUNEL
}

Abstract. It is shown that for an SDE in a Hilbert space, eventual compactness of the driving semigroup together with compact perturbations can be used to establish the existence of an invariant measure. The result is applied to stochastic functional differential equations and the heat equation perturbed by delay and noise, which are both shown to be driven by an eventually compact semigroup.

\section{Introduction}

We consider here infinite-dimensional diffusions in a Hilbert space $H$ described by the differential equation

$$
\left\{\begin{array}{l}
\mathrm{d} X(t)=[A X(t)+F(X(t))] \mathrm{d} t+G(X(t)) \mathrm{d} W(t), \quad t \geq 0 \\
X(0)=x
\end{array}\right.
$$

with $A$ the generator of a strongly continuous semigroup, $F$ and $G$ Lipschitz functions and $W$ a Wiener process.

For many choices of $A, F$ and $G$ it is impossible to obtain the exact solution of such an equation. Therefore, it is important to establish qualititative properties of the solution on the basis of information on $A, F, G$ and $W$.

One of these qualitative properties is the existence of an invariant measure: under what conditions does a measure $\mu$ on $H$ exist such that if the initial condition $x$ has distribution $\mu$, we have that $X(t)$ has distribution $\mu$ for all $t \geq 0$.

Often a compactness argument (Krylov-Bogoliubov) is used to establish the existence of an invariant measure. In finite dimensions it suffices to show that the solutions of (1.1) are bounded in probability. In infinite dimensions, due to the absence of local compactness, we need to exploit compactness properties of the solutions of the stochastic differential equation.

Mathematics Subject Classification (2000): Primary 60H10, Secondary 47D06

Keywords: Delay equation, Eventually compact semigroup, Invariant measure, Reaction diffusion equation, Stochastic evolution equation, Tightness.

O. van Gaans acknowledges the financial support by a "Vidi subsidie" (639.032.510) of the Netherlands Organisation for Scientific Research (NWO). 
It has been shown [3] that a suitable criterion is that $A$ generates a compact semigroup. Together with solutions bounded in probability this suffices to prove the existence of an invariant measure. Another approach is taken in [14], based on hyperbolicity of the driving semigroup and small Lipschitz coefficients of the perturbations.

The result obtained by compactness of the semigroup leads immediately to the question whether eventual compactness of the semigroup can be used to establish existence of an invariant measure. This is an interesting question because, for example, delay differential equations, when put in an infinite-dimensional framework, possess this property (see [6]). Also in the theory of structured population equations eventually compact semigroups appear (see e.g. [7] and [8], Section VI.1). In [4] it is conjectured that eventual compactness should be a sufficient criterion for the existence of an invariant measure.

In this paper, we show that eventual compactness of the semigroup, together with compact factorizations of the perturbations $F$ and $G$, can indeed be used to establish the existence of an invariant measure (Sect. 2). As an example, the result is applied to a stochastic functional differential equation and the currently very active (see eg. [10]) field of reaction diffusion equations perturbed by delayed feedback and noise (Sect. 3). In Appendix A the eventual compactness of the delay semigroup, applied to partial differential equations, is established. This is a generalization of the well-known fact that ordinary delay differential equations are described by eventually compact semigroups.

\section{Main result}

Throughout this section, we will assume that the following hypothesis holds:

HYPOTHESIS 2.1. (i) $H$ and $U$ are separable Hilbert spaces and $E_{1}$ and $E_{2}$ are Banach spaces;

(i) A is the generator of a strongly continuous, eventually compact semigroup $(S(t))$ on $H$; we assume without loss of generality that $S(t)$ is compact for $t \geq 1$;

(ii) $F: H \rightarrow H$ is globally Lipschitz and admits a factorization $F=C_{1} \circ \Phi$, where $C_{1} \in L\left(E_{1} ; H\right)$ is compact and $\Phi: H \rightarrow E_{1}$;

(iii) $G: H \rightarrow L_{H S}(U ; H)$ is globally Lipschitz and admits a factorization $G(x)=$ $C_{2} \Psi(x), x \in H$, where $C_{2} \in L\left(E_{2} ; H\right)$ is compact, and $\Psi: H \rightarrow L_{H S}(U$; $\left.E_{2}\right){ }^{1}$

(iv) $W$ is a cylindrical Wiener process in $U$;

(v) $(X(t, x))_{t \geq 0, x \in H}$ is the unique mild solution [4, Theorem 5.3.1] of the stochastic differential equation

$$
\left\{\begin{array}{l}
\mathrm{d} X(t)=(A X(t)+F(X)) \mathrm{d} t+G(X) \mathrm{d} W(t) \\
X(0)=x, \quad x \in H
\end{array}\right.
$$

\footnotetext{
${ }^{1}$ Here $L_{H S}(U ; H)$ denotes the space of Hilbert-Schmidt operators from $U$ into $H$.
} 
(vi) For all $x \in H$ and $\varepsilon>0$, there exists $R>0$ such that for all $T \geq 1$,

$$
\frac{1}{T} \int_{0}^{T} \mathbb{P}(|X(t, x)| \geq R) \mathrm{d} t<\varepsilon .
$$

Under these assumptions, we will establish the existence of an invariant measure. First we need a couple of lemmas.

LEMMA 2.2. Let $E_{1}, E_{2}$ be Banach spaces. Let $(T(t))$ be a strongly continuous semigroup acting on $E_{1}$ and suppose $C \in L\left(E_{2} ; E_{1}\right)$ is compact. Let

$$
G f:=\int_{0}^{1} T(1-s) C f(s) \mathrm{d} s, \quad f \in L^{p}\left([0,1] ; E_{2}\right),
$$

with $p \geq 2$. Then $G \in L\left(L^{p}\left([0,1] ; E_{2}\right) ; E_{1}\right)$ is compact.

Proof. Consider the set

$$
V=\left\{T(t) C k: t \in[0,1], k \in E_{2},|k| \leq 1\right\}
$$

We will show that $V$ is relatively compact. Indeed, let $\left(v_{n}\right)$ be a sequence in $V$. There exist sequences $\left(t_{n}\right) \subset[0,1],\left(x_{n}\right) \subset E_{2}$, with $\left|x_{n}\right| \leq 1, n \in \mathbb{N}$, such that

$$
v_{n}=T\left(t_{n}\right) C x_{n}, \quad n \in \mathbb{N} .
$$

Since $C$ is compact, there exists a subsequence $\left(x_{n_{k}}\right)$ of $\left(x_{n}\right)$ such that $C x_{n_{k}} \rightarrow y$ for some $y \in E_{1},|y| \leq\|C\|$. Since $[0,1]$ is compact, by strong continuity of $(T(t))$, there exists a further subsequence $\left(t_{n_{k_{l}}}\right)$ of $\left(t_{n_{k}}\right)$ such that $T\left(t_{n_{k_{l}}}\right) y \rightarrow z$ with $z \in T([0,1]) y$.

Now

$$
\begin{aligned}
\left|T\left(t_{n_{k_{l}}}\right) C x_{n_{k_{l}}}-z\right| & \leq\left|T\left(t_{n_{k_{l}}}\right) C x_{n_{k_{l}}}-T\left(t_{n_{k_{l}}}\right) y\right|+\left|T\left(t_{n_{k_{l}}}\right) y-z\right| \\
& \leq|| T\left(t_{n_{k_{l}}}\right)||\left|C x_{n_{k_{l}}}-y\right|+\left|T\left(t_{n_{k_{l}}}\right) y-z\right| \\
& \leq m\left|C x_{n_{k_{l}}}-y\right|+\left|T\left(t_{n_{k_{l}}}\right) y-z\right| \rightarrow 0
\end{aligned}
$$

as $l \rightarrow \infty$. Here $m=\sup _{t \in[0,1]}\|T(t)\|$.

So $V$ is relatively compact and therefore its closed convex hull $K$ is compact [12, Theorem 3.25].

Now define a positive measure on $[0,1]$ by

$$
\mu_{f}(\mathrm{~d} s):=|f(s)| \mathrm{d} s .
$$

Note that $\mu_{f}$ is a finite measure since, by Jensen,

$$
\mu_{f}([0,1])=\int_{0}^{1}|f(s)| \mathrm{d} s \leq\left(\int_{0}^{1}|f(s)|^{p} \mathrm{~d} s\right)^{\frac{1}{p}} .
$$


Now

$$
G f=\int_{0}^{1} T(1-s) C \frac{f(s)}{|f(s)|} \mu_{f}(\mathrm{~d} s),
$$

is an integral over positive, finite measure with the integrand assuming values in the convex set $K$, so

$$
G f \in \mu_{f}([0,1]) K=\|f\|_{L^{p}\left([0,1] ; E_{2}\right)} K .
$$

We will need the following lemma.

LEMMA 2.3. Let $H$ be a separable Hilbert space. Let $K \subset H$ be compact. Then there exists a compact, self-adjoint, strictly positive definite operator $T \in L(H)$ such that

$$
K \subset\{T x:|x| \leq 1\}
$$

Proof. The proof is from [2, Example 3.8.13(ii)]. Assume for simplicity we deal with $\ell^{2}$ and there exists a non-zero $x \in K$.

CLAIM.

$$
\lim _{n \rightarrow \infty} \sup _{x \in K} \sum_{i=n}^{\infty} x_{i}^{2}=0 .
$$

Proof of claim. Suppose there exists a $\delta>0$ such that for all $n \in \mathbb{N}$, there exists $x^{n} \in K$ such that

$$
\sum_{i=n}^{\infty}\left(x_{i}^{n}\right)^{2} \geq \delta .
$$

Now for fixed $n$ pick such an $x^{n}$ and $m \in \mathbb{N}$ such that

$$
\sum_{i=m}^{\infty}\left(x_{i}^{n}\right)^{2}<\delta / 2 .
$$

Furthermore, pick $x^{m}$ such that

$$
\sum_{i=m}^{\infty}\left(x_{i}^{m}\right)^{2} \geq \delta
$$

Then

$$
\left\|x^{n}-x^{m}\right\|_{\ell^{2}}^{2} \geq\left\|\left(x^{n}-x^{m}\right) \mathbb{1}_{\{m, m+1, \ldots\}}\right\|_{\ell^{2}}^{2}>\delta / 2 .
$$

So the sequence $\left(x^{n}\right)$ does not have a Cauchy subsequence. Hence $K$ is not compact, which proves the claim. 
So we can find an increasing sequence $\left(N_{n}\right)_{n=1}^{\infty}$ such that

$$
\sum_{i=N_{n}}^{\infty} x_{i}^{2} \leq 4^{-n} \text { for all } x \in K
$$

Let $t_{i}>0, t_{i}^{2}:=2^{-n+1}$ for $N_{n} \leq i<N_{n+1}, n \in \mathbb{N}$, and $t_{i}^{2}:=2 \sup _{x \in K}\|x\|_{\ell^{2}}^{2}$ for $1 \leq i<N_{1}$. Define $T \in L(H)$ by $(T x)_{i}:=t_{i} x_{i}$.

Since $t_{n} \downarrow 0$, we see that $T$ is compact. Furthermore, if $x \in K$, then let $y=$ $\left(y_{i}\right)_{i=1}^{\infty} \in \ell^{2}$ with $y_{i}=\frac{x_{i}}{t_{i}}, i \in \mathbb{N}$. Then $T y=x$, and

$$
\sum_{i=1}^{\infty} y_{i}^{2}=\sum_{i=1}^{\infty}\left(\frac{x_{i}}{t_{i}}\right)^{2}=\sum_{i=1}^{N_{1}-1}\left(\frac{x_{i}}{t_{i}}\right)^{2}+\sum_{n=1}^{\infty} \sum_{i=N_{n}}^{N_{n+1}-1}\left(\frac{x_{i}}{t_{i}}\right)^{2}
$$

with

$$
\sum_{i=N_{n}}^{N_{n+1}-1}\left(\frac{x_{i}}{t_{i}}\right)^{2}=2^{n-1} \sum_{i=N_{n}}^{N_{n+1}-1} x_{i}^{2} \leq 2^{-n-1} \text { and } \sum_{i=1}^{N_{1}-1}\left(\frac{x_{i}}{t_{i}}\right)^{2} \leq \frac{1}{2} .
$$

We may conclude that

$$
\sum_{i=1}^{\infty} y_{i}^{2} \leq \frac{1}{2}+\sum_{n=1}^{\infty} 2^{-n-1}=1
$$

so $y \in B(0,1)$. It follows that $K \subset T(B(0,1))$.

Now consider, for $x \in H$, the stochastic variable

$$
Y_{x}:=\int_{0}^{1} S(1-s) G(X(s, x)) \mathrm{d} W(s) .
$$

LEMMA 2.4. For all $\varepsilon>0$ and $r>0$, there exists a compact $K(r, \varepsilon) \subset H$ such that

$$
\mathbb{P}\left(Y_{x} \in K(r, \varepsilon)\right)>1-\varepsilon
$$

for all $|x| \leq r$.

Proof. Recall the factorization $G=C_{2} \Psi$ through the Banach space $E_{2}$ from Hypothesis 2.1 with $C_{2}$ compact. In the proof of Lemma 2.2, it is shown that if we let

$$
V=\left\{S(t) C_{2} k: t \in[0,1], k \in E_{2},|k| \leq 1\right\},
$$

and $K$ the closed convex hull of $V$, then $K$ is compact. Let $T \in L(H)$, compact, be as given by Lemma 2.3, so $K \subset T(B(0,1))$ and since $T$ is injective, $V \subset K \subset \mathfrak{D}\left(T^{-1}\right)$. 
Let $K(\lambda):=\lambda T(B(0,1))$ for $\lambda>0$, where $B(0,1)$ is the unit ball in $H$.

Note that

$$
\begin{aligned}
Y_{x} & =\int_{0}^{1} S(1-s) C \Psi(X(s, x)) \mathrm{d} W(s)=\int_{0}^{1} T T^{-1} S(1-s) C \Psi(X(s, x)) \mathrm{d} W(s) \\
& =T \int_{0}^{1} T^{-1} S(1-s) C \Psi(X(s, x)) \mathrm{d} W(s) .
\end{aligned}
$$

So

$$
Y_{x} \in K(\lambda) \Leftrightarrow \int_{0}^{1} T^{-1} S(1-s) C \Psi(X(s, x)) \mathrm{d} W(s) \in \lambda B(0,1) .
$$

Hence, using the fact that $T^{-1} S(1-s) C$ is an operator of norm not greater than 1 (by definition of $T$ ),

$$
\begin{aligned}
\mathbb{P}\left(Y_{x} \notin K(\lambda)\right) & \leq \mathbb{P}\left(\int_{0}^{1} T^{-1} S(1-s) C \Psi(X(s, x)) \mathrm{d} W(s) \notin \lambda B(0,1)\right) \\
& \leq \frac{1}{\lambda^{2}} \mathbb{E}\left[\left|\int_{0}^{1} T^{-1} S(1-s) C \Psi(X(s, x)) \mathrm{d} W(s)\right|^{2}\right] \\
& =\frac{1}{\lambda^{2}} \mathbb{E}\left[\int_{0}^{1}\left|T^{-1} S(1-s) C \Psi(X(s, x))\right|_{H S}^{2} \mathrm{~d} s\right] \\
& \leq \frac{c_{1}}{\lambda^{2}} \mathbb{E}\left[\int_{0}^{1}|\Psi(X(s, x))|_{H S}^{2} \mathrm{~d} s\right] \leq \frac{c_{2}}{\lambda^{2}}\left(1+|x|^{2}\right),
\end{aligned}
$$

for some constants $c_{1}, c_{2}>0$, and where we used [4], Theorem 5.3.1 in the last step. Now pick $\lambda$ large enough such that

$$
\frac{c_{2}}{\lambda^{2}}\left(1+r^{2}\right)<\varepsilon
$$

LEMMA 2.5. Suppose Hypothesis 2.1 is satisfied. For any $\varepsilon>0$ and $r>0$ there exists a compact $K(r, \varepsilon) \subset H$ such that

$$
\mathbb{P}(X(1, x) \in K(r, \varepsilon)) \geq 1-\varepsilon \text { for all } x \in H \text { with }|x| \leq r .
$$

Proof. Note that

$$
X(1, x)=S(1) x+\int_{0}^{1} S(1-s) F(X(s, x)) \mathrm{d} s+\int_{0}^{1} S(1-s) G(X(s, x)) \mathrm{d} W(s) .
$$

We treat the three terms separately.

Since $S(1)$ is a compact operator, for any $r>0$ there exists a compact set $K_{1}(r)$ such that $S(1) x \in K_{1}(r)$ for all $|x| \leq r$. 
Let $p \geq 2$. From [4, Theorem 5.3.1], it follows that there exists a constant $k>0$ such that

$$
\mathbb{E}\left[\int_{0}^{1}|\Phi(X(s, x))|^{p} \mathrm{~d} s\right] \leq k\left(1+|x|^{p}\right)
$$

Define

$$
f: \Omega \times[0,1] \rightarrow E_{1}, \quad f(t):=\Phi(X(t, x)), \quad t \in[0,1] .
$$

Then for $\lambda>0$,

$$
\mathbb{P}\left(|f|_{L^{p}\left(0,1 ; E_{1}\right)}>\lambda\right) \leq \frac{1}{\lambda^{p}} \mathbb{E}\left[|f|_{L^{p}\left(0,1 ; E_{1}\right)}^{p}\right] \leq \frac{k}{\lambda^{p}}\left(1+|x|^{p}\right) \leq \frac{k}{\lambda^{p}}\left(1+r^{p}\right) .
$$

Pick

$$
\lambda:=\left(\frac{2 k}{\varepsilon}\left(1+r^{p}\right)\right)^{1 / p}
$$

so that

$$
\mathbb{P}\left(|f|_{L^{p}\left(0,1 ; E_{1}\right)}>\lambda\right) \leq \varepsilon / 2 .
$$

Hence, by Lemma 2.2 there exists a compact set $K_{2}(\lambda)=K_{2}(r, \varepsilon)$ such that

$$
\mathbb{P}\left(\int_{0}^{1} S(1-s) F(X(s, x)) \mathrm{d} s \in K_{2}(r, \varepsilon)\right)>1-\varepsilon / 2 .
$$

By Lemma 2.4, there exists a compact set $K_{3}(r, \varepsilon)$ such that

$$
\mathbb{P}\left(\int_{0}^{1} S(1-s) G(X(s, x)) \mathrm{d} s \in K_{3}(r, \varepsilon)\right)>1-\varepsilon / 2 .
$$

We may conclude that

$$
\mathbb{P}\left(X(1, x) \in K_{1}(r)+K_{2}(r, \varepsilon)+K_{3}(r, \varepsilon)\right) \geq 1-\varepsilon .
$$

THEOREM 2.6. Suppose Hypothesis 2.1 is satisfied. Then there exists an invariant measure for $(X(t, x))_{t \geq 0}$.

Proof. The proof is analogous to the proof of [4, Theorem 6.1.2].

Let $K(r, \varepsilon)$ as in Lemma 2.5. For $t>1$, using Markov transition probabilities $\left(p_{t}(x, \mathrm{~d} y)\right)$,

$$
\begin{aligned}
& \mathbb{P}(X(t, x) \in K(r, \varepsilon))=\mathbb{E}\left[p_{1}(X(t-1, x), K(r, \varepsilon))\right] \\
& \quad \geq \mathbb{E}\left[p_{1}(X(t-1, x), K(r, \varepsilon)) \mathbb{1}_{\{|X(t-1, x)| \leq r\}}\right] .
\end{aligned}
$$


By Lemma 2.5,

$$
\mathbb{P}(X(t, x) \in K(r, \varepsilon)) \geq(1-\varepsilon) \mathbb{P}(|X(t-1, x)| \leq r),
$$

so

$$
\frac{1}{T} \int_{1}^{T+1} \mathbb{P}(X(t, x) \in K(r, \varepsilon)) \mathrm{d} t \geq \frac{1-\varepsilon}{T} \int_{0}^{T} \mathbb{P}(|X(t, x)| \leq r) \mathrm{d} t .
$$

Now, using Hypothesis 2.1, (vii), take $r$ large enough and $\varepsilon$ small enough, to see that the family

$$
\frac{1}{T} \int_{1}^{T+1} p_{t}(x, \cdot) \mathrm{d} t, \quad T \geq 1,
$$

is tight. By Krylov-Bogoliubov there exists an invariant measure.

It is currently not known to us whether an invariant measure always exists if $F$ and $G$ are only Lipschitz without a factorization property as in Hypothesis 2.1 (ii) and (iii).

\section{Example: stochastic evolutions with delay}

Evolutions with delayed dependence have been studied for some time now. One can think of both ordinary differential equations and partial differential equations, perturbed by a dependence on the 'past' of the process, leading to functional differential equations (see [5,6,9]) and partial functional differential equations (see [13]), respectively. The latter class has attracted a lot of research activity recently, see for example [10].

Can we establish the existence of an invariant measure for such evolutions, perturbed by noise? In order to answer this question, we first present the abstract framework in the style of [1].

Let $X, Z$ be a Banach spaces. Consider the abstract differential equation with delay

$$
\left\{\begin{array}{l}
\frac{\mathrm{d}}{\mathrm{d} t} u(t)=B u(t)+\Phi u_{t}, \quad t>0 \\
u(0)=x \\
u_{0}=f
\end{array}\right.
$$

where

(i) $x \in X$;

(ii) $B$ the generator of a strongly continuous semigroup $(S(t))$ in $X$;

(iii) $\mathfrak{D}(B) \stackrel{d}{\hookrightarrow} Z \stackrel{d}{\hookrightarrow} X ;{ }^{2}$

(iv) $f \in L^{p}([-1,0]$; $Z), 1 \leq p<\infty$;

${ }^{2}$ Here $\stackrel{d}{\hookrightarrow}$ denotes continuous and dense embedding. 
(v) $\Phi: W^{1, p}([-1,0] ; Z) \rightarrow X$ a bounded linear operator ${ }^{3}$

(vi) $u:[-1, \infty) \rightarrow X$ and for $t \geq 0, u_{t}:[-1,0] \rightarrow X$ is defined by $u_{t}(\sigma)=$ $u(t+\sigma), \sigma \in[-1,0]$.

A classical solution of (3.1) is a function $u:[-1, \infty) \rightarrow X$ that satisfies

(i) $u \in C([-1, \infty) ; X) \cap C^{1}([0, \infty) ; X)$;

(ii) $u(t) \in \mathfrak{D}(B)$ and $u_{t} \in W^{1, p}([-1,0] ; Z)$ for all $t \geq 0$;

(iii) $u$ satisfies (3.1) for all $t \geq 0$.

To employ a semigroup approach we introduce the Banach space

$$
\mathcal{E}^{p}:=X \times L^{p}([-1,0] ; Z),
$$

and the closed, densely defined operator in $\mathcal{E}^{p}$,

$$
A:=\left[\begin{array}{cc}
B & \Phi \\
0 & \frac{\mathrm{d}}{\mathrm{d} \sigma}
\end{array}\right], \quad \mathfrak{D}(A)=\left\{\left(\begin{array}{l}
x \\
f
\end{array}\right) \in X \times W^{1, p}([-1,0] ; Z): f(0)=x\right\} .
$$

The Eq. (3.1) is called wellposed if for all $(x, f) \in \mathfrak{D}(A)$, there exists a unique classical solution of (3.1) that depends continuously on the initial data (in the sense of uniform convergence on compact intervals).

It is shown in [1] that $A$ generates a strongly continuous semigroup in $\mathcal{E}^{p}$ if and only if (3.1) is wellposed. Furthermore, sufficient conditions on $\Phi$ are given for this to be the case:

HYPOTHESIS 3.1. Let $S_{t}: X \rightarrow L^{p}([-1,0] ; Z)$ be defined by

$$
\left(S_{t} x\right)(\tau):= \begin{cases}S(t+\tau) x & \text { if }-t<\tau \leq 0, \\ 0 & \text { if }-1 \leq \tau \leq-t, \quad t \geq 0 .\end{cases}
$$

Let $\left(T_{0}(t)\right)_{t \geq 0}$ be the nilpotent left shift semigroup on $L^{p}([-1,0] ; Z)$. Assume that there exists a function $q:[0, \infty) \rightarrow[0, \infty)$ with $\lim _{t \downarrow 0} q(t)=0$, such that

$$
\int_{0}^{t}\left\|\Phi\left(S_{r} x+T_{0}(r) f\right)\right\| \mathrm{d} r \leq q(t)\left\|\left(\begin{array}{l}
x \\
f
\end{array}\right)\right\|
$$

for all $t>0$ and $\left(\begin{array}{l}x \\ f\end{array}\right) \in \mathfrak{D}(A)$. Furthermore, suppose that either

(A) $Z=X$ or

(B) (i) $(B, \mathfrak{D}(B))$ generates an analytic semigroup $(S(t))_{t \geq 0}$ on $X$, and

(ii) for some $\delta>\omega_{0}(B)$ there exists $\vartheta<\frac{1}{p}$ such that

$$
\mathfrak{D}\left((-B+\delta I)^{\vartheta}\right) \stackrel{d}{\hookrightarrow} Z \stackrel{d}{\hookrightarrow} X
$$

THEOREM 3.2. Assume Hypothesis 3.1 holds. Then $(A, \mathfrak{D}(A))$ is the generator of a strongly continuous semigroup $(T(t))_{t \geq 0}$ on $\mathcal{E}^{p}$.

\footnotetext{
${ }^{3}$ Here $W^{k, p}(U ; V)$ denotes the Sobolev space consisting of equivalence classes of functions mapping from $U$ into $V$ with partial derivatives up to and including $k$ th order in $L^{p}(U ; V)$.
} 
Proof. See [1, Theorem 3.26 and Theorem 3.34].

EXAMPLE. Let $\Phi: C([-1,0] ; Z) \rightarrow X$ be given by

$$
\Phi(f):=\int_{-1}^{0} \mathrm{~d} \eta f
$$

where $\eta:[-1,0] \rightarrow L(Z ; X)$ is of bounded variation.

Suppose either that $Z=X$ or that $(B, \mathfrak{D}(B))$ satisfies the assumptions (B-i) and (B-ii) of Hypothesis 3.1. Then the conditions of Theorem 3.2 are satisfied and hence $(A, \mathfrak{D}(A))$ generates a strongly continuous semigroup (see [1, Theorem 3.29 and Theorem 3.35]).

By the following theorem, proven in Sect. A, we see that in many cases $A$ generates an eventually compact semigroup.

THEOREM 3.3. Suppose Hypothesis 3.1 holds. Furthermore, suppose $(S(t))_{t \geq 0}$ is immediately compact. Then $(T(t))_{t \geq 0}$ is compact for all $t>1$. If $X$ is finite dimensional, then $(T(t))_{t \geq 0}$ is also compact at $t=1$.

\subsection{Example (Functional differential equations)}

A relatively easy case is now the example of a functional differential equation perturbed by noise. In the framework introduced above, let $X=Z=\mathbb{R}^{d}, B \in L\left(\mathbb{R}^{d}\right)$ and $\Phi(f)=\int_{-1}^{0} \mathrm{~d} \eta f$, with $\eta:[-1,0] \rightarrow L\left(\mathbb{R}^{d}\right)$ of bounded variation.

As a special case, we can take $\eta(\sigma)=\sum_{i=1}^{n} H\left(\sigma-\theta_{i}\right) B_{i}$, where $H$ denotes the Heaviside step function, $\theta_{i} \in[-1,0]$, and $B_{i} \in L\left(\mathbb{R}^{d}\right), i=1, \ldots, n$. Then (3.1) becomes the delay differential equation

$$
\frac{\mathrm{d} u}{\mathrm{~d} t}=B u(t)+\sum_{i=1}^{n} B_{i} u\left(t-\theta_{i}\right)
$$

We can perturb the functional differential equation by noise to obtain a stochastic functional differential equation of the form

$$
\mathrm{d} u=\left[B u(t)+\int_{-1}^{0} \mathrm{~d} \eta u_{t}+\varphi\left(u(t), u_{t}\right)\right] \mathrm{d} t+\psi\left(u(t), u_{t}\right) \mathrm{d} W(t), \quad t \geq 0,
$$

where $\varphi: \mathcal{E}^{2} \rightarrow \mathbb{R}^{d}, \psi: \mathcal{E}^{2} \rightarrow L\left(\mathbb{R}^{m} ; \mathbb{R}^{d}\right)$ are Lipschitz, and $(W(t))_{t \geq 0}$ is an $m$-dimensional standard Brownian motion.

If we define $F: \mathcal{E}^{2} \rightarrow \mathcal{E}^{2}$ and $G: \mathcal{E}^{2} \rightarrow L\left(\mathbb{R}^{m} ; \mathcal{E}^{2}\right)$ by

$$
F\left(\left[\begin{array}{c}
v \\
w
\end{array}\right]\right):=\left[\begin{array}{c}
\varphi(v, w) \\
0
\end{array}\right], \quad G\left(\left[\begin{array}{c}
v \\
w
\end{array}\right]\right):=\left[\begin{array}{c}
\psi(v, w) \\
0
\end{array}\right], \quad\left[\begin{array}{c}
v \\
w
\end{array}\right] \in \mathcal{E}^{2},
$$

and $A$ as in (3.2), then we arrive in the framework of Eq. (1.1) with as state space $H=\mathcal{E}^{2}$. 
Since $F$ and $G$ map into finite dimensional subspaces of $\mathcal{E}^{2}$, they clearly admit a compact factorization as meant in Hypothesis 2.1. By Theorem 3.3, A generates an eventually compact semigroup. Hence all conditions of Hypothesis 2.1 are satisfied, except possibly condition (vi), boundedness in probability. ${ }^{4}$ If this condition is also satisfied, by Theorem 2.6 we have established the existence of an invariant measure for (3.3) on the state space $\mathcal{E}^{2}$.

\subsection{Example}

Reaction diffusion equations with delayed nonlocal reaction terms are a topic of active research in the study of biological invasion and disease spread. Can we establish the existence of an invariant measure if we add randomness to such a system? As an example, we set out to answer this question for an equation similar to one encountered in e.g. [10].

Consider the reaction diffusion equation with delay and noise

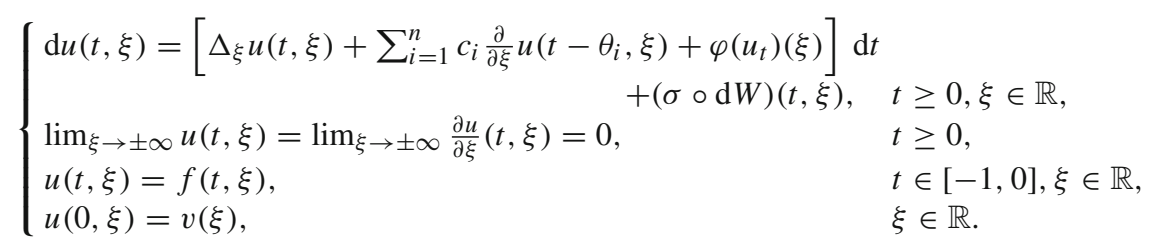

with

(i) delay parameters $c_{i} \in \mathbb{R}, \theta_{i} \in[-1,0], i=1, \ldots, n$,

(ii) initial conditions $f \in L^{2}\left([-1,0] ; W^{1,2}(\mathbb{R})\right)$ and $v \in L^{2}(\mathbb{R})$,

(iii) Lipschitz reaction term $\varphi: L^{2}\left([-1,0] ; W^{1,2}(\mathbb{R})\right) \rightarrow L^{2}(\mathbb{R})$ (possibly nonlinear and/or non-local),

(iv) $u_{t} \in L^{2}\left([-1,0] ; W^{1,2}(\mathbb{R})\right)$ denoting the segment process defined by $u_{t}(\theta)=$ $u(t+\theta)$ for $\theta \in[-1,0], t \geq 0$,

(v) $(W(t))_{t \geq 0}$ a cylindrical Brownian motion in some Hilbert space $U$,

(vi) noise factor $\sigma \in L_{H S}\left(U ; W^{1,2}(\mathbb{R})\right)$.

We can employ the semigroup approach discussed before by setting $X:=L^{2}(\mathbb{R})$, $Z:=W^{1,2}(\mathbb{R})$, as state space the Hilbert space $\mathcal{E}^{2}=X \times L^{2}([-1,0] ; Z)$, with $A$ as defined in (3.2), with

$$
B:=\Delta, \quad \mathfrak{D}(B)=\left\{v \in W^{2,2}(\mathbb{R}): \lim _{\xi \rightarrow \pm \infty} v(\xi)=0, \lim _{\xi \rightarrow \pm \infty} \frac{\partial v}{\partial \xi}(\xi)=0\right\}
$$

and

$$
\Phi(w):=\sum_{i=1}^{n} c_{i} \frac{\partial}{\partial \xi} w\left(t-\theta_{i}, \xi\right), \quad w \in L^{2}\left([-1,0] ; W^{1,2}(\mathbb{R})\right)
$$

\footnotetext{
${ }^{4}$ Boundedness in probability has to be established for individual cases, for example, by posing dissipativity conditions on $A, F$ and $G$, see [3].
} 
Then $A$ is of the form described in Example 3. Since $B$ generates an immediately compact semigroup (see for example [8, Exercise II.4.30(4)]), it follows from Theorem 3.3 that $A$ generates an eventually compact semigroup.

Furthermore, let

$$
F\left(\left[\begin{array}{c}
v \\
w
\end{array}\right]\right):=\left[\begin{array}{c}
\varphi(w) \\
0
\end{array}\right], \quad\left[\begin{array}{c}
v \\
w
\end{array}\right] \in \mathcal{E}^{2}, \quad \text { and } \quad G(\cdot):=\left[\begin{array}{c}
\sigma \\
0
\end{array}\right] \quad \text { on } \mathcal{E}^{2}
$$

Then (3.4) is described by (1.1) in the state space $H=\mathcal{E}^{2}$.

It remains to impose conditions on the nonlinear term $F$. Let us require, for example, that $\varphi: L^{2}\left([-1,0] ; W^{1,2}(\mathbb{R})\right) \rightarrow L^{2}(\mathbb{R})$ is of the form

$$
\varphi(w):=(g \circ h)(w)
$$

with $g: W^{1,2}(\mathbb{R}) \rightarrow W^{1,2}(\mathbb{R})$ (possibly the identity mapping), and $h$ defined by

$$
h(w)(\xi):=\int_{-1}^{0} \int_{\mathbb{R}} k(\eta, \sigma) \psi(w(\sigma, \xi-\eta)) \mathrm{d} \eta \mathrm{d} \sigma, \quad \xi \in \mathbb{R}
$$

where $\psi \in W^{1, \infty}(\mathbb{R})$ with $|\psi(\zeta)| \leq\|\dot{\psi}\|_{\infty}|\zeta|, \zeta \in \mathbb{R}$, and $k \in L^{1}\left(\mathbb{R} ; L^{2}([-1,0])\right)$. We will now verify that in this case

$$
\varphi: L^{2}\left([-1,0] ; W^{1,2}(\mathbb{R})\right) \rightarrow W^{1,2}(\mathbb{R})
$$

Indeed, using Fubini, Cauchy-Schwarz and Young's inequality for convolutions,

$$
\begin{aligned}
& \int_{\mathbb{R}}|h(w)(\xi)|^{2} \mathrm{~d} \xi \\
& =\iint_{\mathbb{R}}\left|\int_{-1}^{0} \int_{\mathbb{R}} k(\eta, \sigma) \psi(w(\sigma, \xi-\eta)) \mathrm{d} \eta \mathrm{d} \sigma\right|^{2} \mathrm{~d} \xi \\
& \leq\|\dot{\psi}\|_{\infty}^{2} \int_{\mathbb{R}}\left(\int_{-1}^{0}\left|\int_{\mathbb{R}}\right| k(\eta, \sigma) w(\sigma, \xi-\eta) \mid \mathrm{d} \eta \mathrm{d} \sigma\right)^{2} \mathrm{~d} \xi \\
& \leq\|\dot{\psi}\|_{\infty}^{2} \int_{\mathbb{R}}\left(\int_{\mathbb{R}}\|k(\eta, \cdot)\|_{L^{2}([-1,0])}\|w(\cdot, \xi-\eta)\|_{L^{2}([-1,0])} \mathrm{d} \eta\right)^{2} \mathrm{~d} \xi \\
& \leq\left(\|\dot{\psi}\|_{\infty}\|k\|_{L^{1}\left(\mathbb{R} ; L^{2}([-1,0])\right)}\|w\|_{L^{2}\left([-1,0] ; L^{2}(\mathbb{R})\right)}\right)^{2} .
\end{aligned}
$$


Furthermore, using the same classic inequalities,

$$
\begin{aligned}
\int_{\mathbb{R}} & \left|\frac{\partial}{\partial \xi} h(w)(\xi)\right|^{2} \mathrm{~d} \xi \\
& =\int\left|\int_{\mathbb{R}} \int_{-1} k(\eta, \sigma) \frac{\partial}{\partial \xi} \psi(w(\sigma, \xi-\eta)) \mathrm{d} \eta \mathrm{d} \sigma\right|^{2} \mathrm{~d} \xi \\
& =\int_{\mathbb{R}}\left|\int_{-1} \int_{\mathbb{R}} k(\eta, \sigma) \dot{\psi}(w(\sigma, \xi-\eta)) \frac{\partial}{\partial \xi} w(\sigma, \xi-\eta) \mathrm{d} \eta \mathrm{d} \sigma\right|^{2} \mathrm{~d} \xi \\
\leq & \|\dot{\psi}\|_{\infty}^{2} \int_{\mathbb{R}}\left(\int_{-1}^{0} \int_{\mathbb{R}}\left|k(\eta, \sigma) \frac{\partial}{\partial \xi} w(\sigma, \xi-\eta)\right|^{2} \mathrm{~d} \eta \mathrm{d} \sigma\right)^{\mathrm{d} \xi} \xi \\
\leq & \|\dot{\psi}\|_{\infty}^{2} \int_{\mathbb{R}}\left(\left.\int_{\mathbb{R}}\|k(\eta, \cdot)\|_{L^{2}([-1,0])}|| \frac{\partial}{\partial \xi} w(\cdot, \xi-\eta)\right|_{L^{2}([-1,0])} \mathrm{d} \eta\right)^{2} \mathrm{~d} \xi \\
\leq & \left(\|\dot{\psi}\|_{\infty}\|k\|_{L^{1}\left(\mathbb{R} ; L^{2}([-1,0])\right)}\|w\|_{\left.L^{2}\left([-1,0] ; W^{1,2}(\mathbb{R})\right)\right)^{2} \cdot}\right.
\end{aligned}
$$

So we have $h: L^{2}\left([-1,0] ; L^{2}(\mathbb{R})\right) \rightarrow W^{1,2}(\mathbb{R})$, and therefore (3.5) holds for $\varphi=g \circ h$. Hence in this case we may write (with some abuse of notation) $\varphi=\imath \circ \varphi$, where $\imath: W^{1,2}(\mathbb{R}) \rightarrow L^{2}(\mathbb{R})$ is the canonical embedding of $W^{1,2}(\mathbb{R})$ into $L^{2}(\mathbb{R})$, which is a compact mapping. We conclude that $F$ admits a compact factorization. Note that this carries over to any function $\varphi$ that satisfies (3.5). $G$ admits a compact factorization as well, again using the compact embedding of $W^{1,2}(\mathbb{R})$ into $L^{2}(\mathbb{R})$.

Again, we may conclude from Theorem 2.6 that if the solutions of (3.4) are bounded in probability, an invariant measure exists.

Open Access. This article is distributed under the terms of the Creative Commons Attribution Noncommercial License which permits any noncommercial use, distribution, and reproduction in any medium, provided the original author(s) and source are credited.

\section{Appendix A. Eventual compactness of the delay semigroup}

The purpose of this section is to prove Theorem 3.3. We proceed as in [8, Section VI.6]. We will use the following variant of the Arzelà-Ascoli theorem.

DEFINITION A.1. A subset $\Phi$ of $C(X ; Y)$ is pointwise relatively compact if and only if $\forall x \in X$, the set $\{f(x): f \in \Phi\}$ is relatively compact in $Y$.

THEOREM A.2. (vector valued Arzelà-Ascoli, [11, Theorem 47.1]) Let X be a compact Hausdorff space and $Y$ a metric space. Then a subset $\Phi$ of $C(X ; Y)$ is relatively compact if and only if it is equicontinuous and pointwise relatively compact. 
LEMMA A.3. Suppose $(S(t))_{t \geq 0}$ is immediately compact. Then $R(\lambda, A) T(1)$ is compact for all $\lambda \in \rho(A)$.

Proof. According to Bátkai and Piazzera [1], Proposition 3.19, we have the following expression for the resolvent $R(\lambda, A)$ :

$$
R(\lambda, A)=\left[\begin{array}{cc}
R\left(\lambda, B+\Phi_{\lambda}\right) & R\left(\lambda, B+\Phi_{\lambda}\right) \Phi R\left(\lambda, A_{0}\right) \\
\epsilon_{\lambda} R\left(\lambda, B+\Phi_{\lambda}\right)\left[\epsilon_{\lambda} R\left(\lambda, B+\Phi_{\lambda}\right) \Phi+I\right] R\left(\lambda, A_{0}\right)
\end{array}\right], \quad \lambda \in \rho(A),
$$

where, for $\lambda \in \mathbb{C}, \Phi_{\lambda} \in L(X)$ is given by

$$
\Phi_{\lambda} x:=\Phi\left(\mathrm{e}^{\lambda \cdot} x\right), \quad x \in X
$$

$\epsilon_{\lambda}$ is the function

$$
\epsilon_{\lambda}(s):=\mathrm{e}^{\lambda s}, \quad s \in[-1,0] .
$$

and $A_{0}$ is the generator of the nilpotent left-shift semigroup on $L^{p}([-1,0] ; X)$.

Let

$$
\pi_{1}: X \times L^{p}([-1,0] ; Z) \rightarrow X
$$

and

$$
\pi_{2}: X \times L^{p}([-1,0] ; Z) \rightarrow L^{p}([-1,0] ; Z)
$$

denote the canonical projections on $X$ and $L^{p}([-1,0] ; Z)$, respectively.

Lemma 4.5 and Lemma 4.9 in [1] state that the operator $R\left(\lambda, B+\Phi_{\lambda}\right)$ is compact for all $\lambda \in \rho(A)$. Therefore, using (A.1)

$$
\pi_{1} R(\lambda, A) T(1)=\left[R\left(\lambda, B+\Phi_{\lambda}\right) R\left(\lambda, B+\Phi_{\lambda}\right) \Phi R\left(\lambda, A_{0}\right)\right] T(1)
$$

is compact.

We can therefore restrict our attention to

$$
\pi_{2} R(\lambda, A) T(1): X \times L^{p}([-1,0] ; Z) \rightarrow L^{p}([-1,0] ; Z) .
$$

Denote $\varphi:=\left(\begin{array}{l}x \\ f\end{array}\right)$ where $x \in X$ and $f \in L^{p}([-1,0] ; Z)$. Note that

$$
\frac{\mathrm{d}}{\mathrm{d} \sigma} \pi_{2} R(\lambda, A) T(1) \varphi=\pi_{2} A R(\lambda, A) T(1) \varphi,
$$


Hence, using Hölder, there exists some constant $M>0$ such that for all $t_{0}, t_{1} \in$ $[-1,0]$,

$$
\begin{aligned}
\| \pi_{2} R & R, A) T(1) \varphi\left(t_{1}\right)-\pi_{2} R(\lambda, A) T(1) \varphi\left(t_{0}\right) \|_{Z} \\
= & \left\|\int_{t_{0}}^{t_{1}}\left[\frac{\mathrm{d}}{\mathrm{d} \sigma} \pi_{2} R(\lambda, A) T(1) \varphi\right](\sigma) \mathrm{d} \sigma\right\|_{Z} \\
= & \left\|\int_{t_{0}}^{t_{1}}\left[\pi_{2} A R(\lambda, A) T(1) \varphi\right](\sigma) \mathrm{d} \sigma\right\|_{Z} \\
& \leq \int_{t_{0}}^{t_{1}}\left\|\left[\pi_{2} A R(\lambda, A) T(1) \varphi\right](\sigma)\right\|_{Z} \mathrm{~d} \sigma \\
\leq & \left|t_{1}-t_{0}\right|^{1 / q}\left\|\pi_{2} A R(\lambda, A) T(1) \varphi\right\|_{L^{p}([-1,0] ; Z)} \\
\leq & M\left|t_{1}-t_{0}\right|^{1 / q}\|\varphi\|_{\mathcal{E}^{p}} .
\end{aligned}
$$

Here $q>1$ is such that $\frac{1}{q}+\frac{1}{p}=1$.

So

$$
\mathcal{C}:=\left\{\pi_{2} R(\lambda, A) T(1) \varphi: \varphi \in \mathcal{E}^{p},\|\varphi\|_{\mathcal{E}^{p}} \leq 1\right\} \subset C([-1,0] ; Z)
$$

is equicontinuous.

Furthermore, note that

$$
\begin{aligned}
& {\left[\pi_{2} R(\lambda, A) T(1) \varphi\right](\sigma)} \\
& \left.=\left[\pi_{2} T(1) R(\lambda, A) \varphi\right](\sigma) \quad \text { (commutativity of } R(\lambda, A) \text { and } T(1)\right) \\
& \left.=\left[\pi_{2} T(1+\sigma) R(\lambda, A) \varphi\right](0) \quad \text { (translation property of }(T(t))_{t \geq 0}\right) \\
& =\left[\pi_{2} R(\lambda, A) T(1+\sigma) \varphi\right](0) \quad \text { (commutativity) } \\
& =\pi_{1} R(\lambda, A) T(1+\sigma) \varphi \quad(R(\lambda, A) \text { maps to domain } A) \text {. }
\end{aligned}
$$

Again using (A.1) and the fact that $R\left(\lambda, B+\Phi_{\lambda}\right)$ is compact, we find that $\mathcal{C}$ is pointwise relatively compact. By the vector-valued Arzelà-Ascoli theorem, Theorem A.2, we find that $\mathcal{C}$ is relatively compact in $C([-1,0] ; Z)$ and hence relatively compact in $L^{p}([-1,0] ; Z)$.

From this we conclude that $\pi_{2} R(\lambda, A) T(1)$ is compact and combining this with (A.2), $R(\lambda, A) T(1)$ is compact.

We may now conclude that $(T(t))_{t \geq 0}$ is eventually compact:

THEOREM A.4. Suppose Hypothesis 3.1 holds. Furthermore, suppose $(S(t))_{t \geq 0}$ is immediately compact. Then $(T(t))_{t \geq 0}$ is compact for all $t>1$. If $X$ is finite dimensional, then $(T(t))_{t \geq 0}$ is also compact at $t=1$. 
Proof. By Engel and Nagel [8, Lemma II.4.28], it is sufficient to show that $(T(t))$ is eventually norm continuous for $t>1$, and that $R(\lambda, A) T(1)$ is compact for some $\lambda \in \rho(A)$.

Now by [1, Lemma 4.5], $(T(t))$ is norm continuous for $t>1$ (using that $(S(t))$ is immediately compact and hence immediately norm continuous). Furthermore, by Lemma A.3, $R(\lambda, A) T(1)$ is compact for all $\lambda \in \rho(A)$.

For the finite dimensional case, see [6].

\section{REFERENCES}

[1] A. Bátkai and S. Piazzera. Semigroups for Delay Equations. AK Peters, Ltd., 2005.

[2] V.I. Bogachev. Gaussian Measures. American Mathematical Society, 1998.

[3] G. Da Prato, D. Gatarek, and J. Zabczyk. Invariant measures for semilinear stochastic equations. Stochastic Analysis and Applications, 10(4):387-408, 1992.

[4] G. Da Prato and J. Zabczyk. Ergodicity for Infinite Dimensional Systems. Cambridge University Press, 1996.

[5] M.C. Delfour. The largest class of hereditary systems defining a $C_{0}$ semigroup on the product space. Canad. J. Math., 32(4):969-978, 1980.

[6] O. Diekmann, S.A. Van Gils, S.M. Verduyn Lunel, and H.O. Walther. Delay Equations: Functional-, Complex-, and Nonlinear Analysis. Springer Verlag, 1995.

[7] J.A. Dyson, R. Villella-Bressan, and G.F. Webb. Asynchronous exponential growth in an age structured population of proliferating and quiescent cells. Mathematical Biosciences, 177-178:73-83, 2002.

[8] K.J. Engel and R. Nagel. One-Parameter Semigroups for Linear Evolution Equations. Springer Verlag, 2000.

[9] J. Hale. Theory of functional differential equations. Springer-Verlag, New York, second edition, 1977. Applied Mathematical Sciences, Vol. 3.

[10] W.-T. Li, Z.-C. Wang, and J. Wu. Entire solutions in monostable reaction-diffusion equations with delayed nonlinearity. J. Differential Equations, 245(1):102-129, 2008.

[11] J.R. Munkres. Topology, Second Edition. Prentice-Hall, 2000.

[12] W. Rudin. Functional analysis. International Series in Pure and Applied Mathematics. McGraw-Hill Inc., New York, second edition, 1991.

[13] C. C. Travis and G. F. Webb. Existence and stability for partial functional differential equations. In Dynamical systems (Proc. Internat. Sympos., Brown Univ., Providence, R.I., 1974), Vol. II, pages 147-151. Academic Press, New York, 1976.

[14] O. van Gaans and S.M. Verduyn Lunel. Long Term Behavior Of Dichotomous Stochastic Differential Equations In Hilbert Spaces. Communications in Contemporary Mathematics, 6(3):349-376, 2004.

J. Bierkens, O. van Gaans, S. V. Lunel

Mathematical Institute,

PO Box 9512,

2300 RA Leiden,

The Netherlands

E-mail: joris@math.leidenuniv.nl

O. van Gaans

E-mail:vangaans@math.leidenuniv.nl

S. V. Lunel

E-mail:verduyn@math.leidenuniv.nl 